\title{
Implementation of a Hybrid Approach to Improve Resident Performance on the American Board of Podiatric Surgery In-training Exam
}

\author{
Christine Miller • Jason Piraino
}

Published online: 2 December 2014

(C) International Association of Medical Science Educators 2014

\begin{abstract}
The American Board of Podiatry Surgery (ABPS) examination is given after completion of residency training. Successfully passing this test is pivotal for the practice of podiatric medicine. Traditionally, preparation for this exam consists of in-person lectures given by surgical faculty and the self-study method of reading podiatric manuals. A new method will be introduced using hybrid modules with corresponding online examinations. The results from this new technique will be compared to previous scores to assess whether or not this method is advantageous.
\end{abstract}

Keywords Podiatric surgery $\cdot$ Hybrid module $\cdot$ Residency training $\cdot$ Examination

Receiving a passing score on the American Board of Podiatric Surgery (ABPS) exam upon the completion of residency training is crucial to the career of practicing podiatrists. The traditional methods of exam preparation consist of in-personal lectures given by faculty and the self-study approach of reading the established podiatric manuals. Additionally, some state podiatric societies offer in-person conference lectures for preparation along with a manual for a significant cost. There is little opportunity to gain experience with online testing since a practice exam is only administered once a year.

A new approach with the goal of improved resident performance via hybrid modules will be implemented. Online instruction and examinations will be added to the preparatory process. There is a shortage of literature regarding the hybrid approach in podiatric surgical and medicine residency training.

Residents will be provided with modules in both podiatric medicine and surgery. The medicine modules will be administered online via asynchronous lectures and synchronous review sessions using WebEx. The three modules will cover the topics of clinical radiology, infectious disease, and internal medicine. An online exam will be administered and scored at the completion of each module to ensure mastery of the subject matter. A 70\% score is the minimum score required to move forward to the next module. Each exam will consist of 25 multiple-choice questions. The three surgery modules (forefoot, rear-foot, and reconstructive techniques) will be conducted in the traditional in-person format and will have corresponding online examinations.

The beneficial aspects of the new approach are that lectures can be replayed, as the in-person sessions are not recorded. The synchronous activities allow for questions to be answered in a small group setting. The added opportunity to take tests online serves as practice for the actual ABPS examination.

The exam scores for this coming year will be compared to those of the past 5 years. Assessment of the new module will be conducted to see if the hybrid model had any effect on the test performance. 